\title{
CONDITIONALITY OF INVERSE SOLUTIONS TO DISCRETISED INTEGRAL EQUATIONS IN GEOID MODELLING FROM LOCAL GRAVITY DATA
}

\author{
R. TENZER ${ }^{1}$, P. NOVÁK 2,3 \\ 1 Faculty of Aerospace Engineering, Physical and Space Geodesy (PSG), Delft University of \\ Technology, Kluyverweg 1, 2629 HS Delft, The Netherlands (R.Tenzer@tudelft.nl) \\ 2 Research Institute of Geodesy, Topography and Cartography, Ondřejov 244, 25165 Czech \\ Republic \\ 3 Department of Mathematics, University of Western Bohemia, 30614 Plzeň, Czech Republic
}

Received: April 23, 2007; Revised: September 21, 2007; Accepted: October 11, 2007

\begin{abstract}
The eigenvalue decomposition technique is used for analysis of conditionality of two alternative solutions for a determination of the geoid from local gravity data. The first solution is based on the standard two-step approach utilising the inverse of the AbelPoisson integral equation (downward continuation) and consequently the Stokes/Hotine integration (gravity inversion). The second solution is based on a single integral that combines the downward continuation and the gravity inversion in one integral equation. Extreme eigenvalues and corresponding condition numbers of matrix operators are investigated to compare the stability of inverse problems of the above-mentioned computational models. To preserve a dominantly diagonal structure of the matrices for inverse solutions, the horizontal positions of the parameterised solution on the geoid and of data points are identical. The numerical experiments using real data reveal that the direct gravity inversion is numerically more stable than the downward continuation procedure in the two-step approach.
\end{abstract}

Key words: geoid, gravity, inverse problem, numerical stability

\section{INTRODUCTION}

The downward continuation of gravity is an ill-posed problem in sense of large highfrequency uncertainties in results of downward continued values due to errors within input gravity data. To obtain stable results, an appropriate regularization should be applied. The regularization techniques of ill-posed problems can be found for instance in Tikhonov (1963), Tikhonov and Arsenin (1977), and Lavrentiev et al. (1986). Methods of regularization associated with the downward continuation procedure were analysed for instance in Sideris and Forsberg (1991), Ilk (1993) and Engels et al. (1993). A different principle of regularization was proposed in Martinec (1996) according to which the 
regularization is to some extent applicable by the spatial discretization of a solution. In particular, the high-frequency part of the solution, determination of which makes the problem unstable, is a priori excluded from the discretised downward continuation model. The optimal regularization is then based on finding the smallest step of data discretisation for which the inverse problem has a stable solution.

Assuming that an implicit incorporation of the Stokes/Hotine integration to the downward continuation of gravity anomalies/disturbances may improve the stability of inverse solutions, an alternative method of computing the disturbing gravity potential has been formulated in Novák (2003) and analysed in Novák et al. (2003) and Alberts and Klees (2004). The direct relation between the observed gravity anomalies/disturbances and the disturbing gravity potential values on the geoid is defined in terms of a single integral equation. The inverse solution to the system of discretised integral equations, the generic form of which is the Fredholm integral equation of the first kind (cf. Arfken, 1985), yields the result in terms of the disturbing gravity potential values parameterised at the geoid directly from the observed gravity anomalies/disturbances.

Despite scepticism among some geodesists, Martinec $(1996,1998)$ and others showed a good performance of the harmonic downward continuation in sense of the numerical stability. Furthermore, results of the numerical analysis in Novák (2003) based on synthetic random noise modelling indicate a good performance of the direct gravity inversion. Following theoretical developments in Novák (2003) and Martinec (1998), the numerical stability of the direct gravity inversion is analysed in this study. The eigenvalue decomposition technique is used for the analysis of the conditionality. The discretised downward continuation of observed gravity and the discretised direct gravity inversion are summarized in Section 2. The eigenvalue decomposition technique is applied in analysing the numerical stability of the inverse solutions in Section 3 with a complementary numerical study and discussion of results in Section 4.

\section{INVERSE SOLUTION FOR THE NEAR-ZONE CONTRIBUTION}

The computation of the near-zone contribution from the local ground and airborne gravity data is realized either by solving the two boundary-value problems involving the downward continuation with the successive Hotine/Stokes integration, or by the direct gravity inversion. Methods of computing the near-zone contributions are summarized in this section. The far-zone contribution not discussed in this study can be estimated from a global geopotential model; see for instance Martinec (1996,1998) and Novák (2000).

The discrete downward continuation of gravity is given by (Bjerhammar, 1962; see also Bjerhammar, 1963,1987)

$$
R \Delta \boldsymbol{g}_{g}^{n z} \cong \mathbf{F} \mathbf{P}^{-1} \mathbf{R}_{t}\left(\Delta \boldsymbol{g}_{t}-\Delta \mathbf{g}_{t}^{f z}\right),
$$

where $\Delta g_{t}$ is the vector of gravity anomalies $\Delta g\left(x_{i}\right)$ observed at the points $\left\{\boldsymbol{x}_{i}: i=1, \ldots, I\right\}$ at/above the Earth's surface. The vector $\Delta \boldsymbol{g}_{t}^{f z}$ of the far-zone contributions to gravity anomalies is subtracted from $\Delta \boldsymbol{g}_{t}$. The solution of Eq.(1) provides 
the vector $\Delta \boldsymbol{g}_{g}^{n z}$ of the near-zone contributions to gravity anomalies $\Delta g\left(\boldsymbol{y}_{i}\right)$ which are parameterised at the geoidal points $\left\{\boldsymbol{y}_{i}: i=1, \ldots, I\right\}$. We assume that the horizontal positions of $\boldsymbol{x}_{i}$ and $\boldsymbol{y}_{i}$ are identical, and these points form the coarse grid with the equalangular step size. The matrix of discretised Abel-Poisson integral equations of Eq.(I.2) is denoted as $\mathbf{P} ; \mathbf{R}_{t}$ is the diagonal matrix of the geocentric radii $\left\{\left|\boldsymbol{x}_{i}\right|: i=1, \ldots, I\right\}$; i.e., $\left\{\mathbf{R}_{t}\right\}_{i, j}=\delta_{i, j}\left|\boldsymbol{x}_{i}\right|$, where $\delta_{i, i}=1$ and otherwise $\delta_{i, j}=0 ; \mathbf{F}$ is the diagonal filter matrix of the solution; and $R$ is the mean radius of the Earth. To unify the size of the near-zone contribution for each equation in the system of Eq.(1), zero values are assigned to all coefficients of the matrix $\mathbf{P}$ which extend the near-zone integration sub-domain, i.e., $\{\mathbf{P}\}_{i, j}=0$ for all $\psi_{i, j}>\psi_{o}$, where $\psi_{o}$ is the maximal spherical angle of the near-zone integration sub-domain. The diagonal elements of the filter matrix $\mathbf{F}$ are formed so that the matrix-vector product of $\mathbf{F}$ and $\mathbf{P}^{-1} \mathbf{R}_{t}\left(\Delta \boldsymbol{g}_{t}-\Delta \boldsymbol{g}_{t}^{f z}\right)$ provides the solution only for the values of the vector $\Delta \boldsymbol{g}_{g}^{n z}$ which comprise entirely the near-zone contribution.

By analogy with Eq.(1), gravity disturbances are downward continued according to

$$
R \boldsymbol{\delta} \boldsymbol{g}_{g}^{n z} \cong \mathbf{F P} \mathbf{P}^{-1} \mathbf{R}_{t}\left(\boldsymbol{\delta} \boldsymbol{g}_{t}-\boldsymbol{\delta} \boldsymbol{g}_{t}^{f z}\right),
$$

where $\delta g_{t}$ is the vector of the gravity disturbances $\delta g\left(x_{i}\right)$ from which the vector of the far-zone contributions $\boldsymbol{\delta} \boldsymbol{g}_{t}^{f z}$ is subtracted, and $\boldsymbol{\delta} \boldsymbol{g}_{g}^{n z}$ is the vector of the near-zone contributions to gravity disturbances $\delta g\left(\boldsymbol{y}_{i}\right)$ on the geoid.

The direct gravity disturbance inversion is given by

$$
\tau_{g}^{n z} \cong \mathbf{F}\left[-\mathbf{K}^{-1} \mathbf{R}_{t}\left(\boldsymbol{\delta} \boldsymbol{g}_{t}-\boldsymbol{\delta} \boldsymbol{g}_{t}^{f z}\right)\right]
$$

where $\tau_{g}^{n z}$ is the vector of the near-zone contributions to disturbing gravity potentials $T\left(\boldsymbol{y}_{i}\right)$. The matrix $\mathbf{K}$ in Eq.(3) is defined as $\mathbf{K}=\mathbf{R}_{t} \partial \mathbf{P} / \partial r$. The direct gravity anomaly inversion reads

$$
\tau_{g}^{n z} \cong \mathbf{F}\left[-\mathbf{M}^{-1} \mathbf{R}_{t}\left(\Delta \boldsymbol{g}_{t}-\Delta \mathbf{g}_{t}^{f z}\right)\right]
$$

with the matrix $\mathbf{M}$ formed by $\mathbf{M}=\mathbf{R}_{t}\left((\partial \mathbf{P} / \partial r)+2 \mathbf{R}_{t}^{-1} \mathbf{P}\right)=\mathbf{K}+2 \mathbf{P}$.

Data interpolation and advanced numerical integration are indispensable in forming the diagonal and near-diagonal elements of the matrices $\mathbf{P}, \mathbf{K}$ and $\mathbf{M}$ due to the behaviour of the kernels $\mathcal{P}, \mathcal{J}$ and $\mathcal{M}$ at the vicinity of the computation point (see Appendix I). The direct matrix inversion can be avoided using iterative methods of solving the large systems of linear equations. An overview of iterative methods can be found for instance in Varga (1962), Young (1972) and Hageman and Young (1981). 


\section{ANALYSIS OF CONDITIONALITY}

To analyse the conditionality of the matrix $\mathbf{M}$, the eigenvalue decomposition technique (e.g., Arfken 1985, Chapter 4.7) can be used. If any eigenvalue $\lambda_{M}$ equals zero, the matrix $\mathbf{M}$ is singular; likewise unstable when $\lambda_{M}$ is very small. Consequently, the conditionality of the matrix $\mathbf{M}$ is classified by the condition number $\boldsymbol{\aleph}_{M}$ defined as $\boldsymbol{\aleph}_{M}=\left\|\mathbf{M}^{-1}\right\| \cdot\|\mathbf{M}\|$, where $\|\mathbf{M}\|$ is a norm of the matrix $\mathbf{M}$. Since $\mathbf{M}$ is a symmetric matrix $\left(\mathbf{M}^{T}=\mathbf{M}\right)$ and thus $\mathbf{M}^{T} \mathbf{M} \equiv \mathbf{M M}^{T}$, the condition number $\aleph_{M}$ can be specified in terms of the Frobenius norm $\|\mathbf{M}\|_{F}^{2}$ (defined as $\|\mathbf{M}\|_{F}^{2}=\operatorname{trace}\left(\mathbf{M}^{*} \mathbf{M}\right)$, where $\mathbf{M}^{*}$ denotes the conjugate transpose of M) as the ratio of the absolute values of the maximal and minimal eigenvalues (Wilkinson 1965, Chapter 2.30), whence

$$
\aleph_{M}=\frac{\left|\max \lambda_{M}\right|}{\left|\min \lambda_{M}\right|} .
$$

$\mathbf{M}$ is singular when the condition number $\boldsymbol{\aleph}_{M}$ is infinite. The upper and lower bounds of the eigenvalues $\lambda_{M}$ of the matrix $\mathbf{M}$ can be estimated according to the Gerschgorin theorem (Wilkinson 1965, Chapter 2.13)

$$
\begin{gathered}
\max \lambda_{M} \leq \max _{i} \sum_{j=1}^{I}\left|\{\mathbf{M}\}_{i, j}\right|, \quad i=1, \ldots, I, \\
\min \lambda_{M} \geq \min _{i}\left(\{\mathbf{M}\}_{i, i}-\sum_{\substack{j=1 \\
(j \neq i)}}^{I}\left|\{\mathbf{M}\}_{i, j}\right|\right) .
\end{gathered}
$$

The upper and lower bounds of the eigenvalues $\lambda_{P}$ of the matrix $\mathbf{P}$ for the downward continuation, see Eqs.(1) and (2), for the full integration domain were estimated in Martinec (1998, Chapter 8.6.1). They read

$$
\max \lambda_{P} \leq \max _{i} \frac{R}{r_{i}}, \quad \min \lambda_{P} \geq \min _{i}\left(1-\frac{2 H_{i}}{\ell\left(r_{i}, \Delta \psi, R\right)}\right),
$$

where $\Delta \psi$ is the discretisation step (at the vicinity of the computation point) with respect to the spherical angle $\psi$, the geocentric radii of the computation points are denoted as $\left\{r_{i}=\left|\boldsymbol{x}_{i}\right|: i=1, \ldots, I\right\} ; \ell=|\boldsymbol{x}-\boldsymbol{y}|$ is the Euclidean spatial distance between the points $\boldsymbol{x}$ and $\boldsymbol{y}$; and $H=r-R$. It can be shown that zero- and first-degree terms of the AbelPoisson kernel, both of a long-wavelength character, have no significant influence on the conditionality of the matrix $\mathbf{P}$ as well as the matrices $\mathbf{K}$ and $\mathbf{M}$. To quantify the influence 


\section{Conditionality of Inverse Solutions to Discretised Integral Equations in Geoid Modelling ...}

of the far-zone effect on the conditionality of the matrix $\mathbf{P}$, the upper and lower bounds of the eigenvalues $\lambda_{P}$ are expressed for an arbitrary near-zone surface integration subdomain defined in terms of the spherical azimuth $\alpha \in\langle 0,2 \pi)$ and the spherical angle $\psi \in\left\langle 0, \psi_{o}\right\rangle$.

Since all elements of the matrix $\mathbf{P}$ are non-negative, $\{\mathbf{P}\}_{i, j} \geq 0$, the following identity holds:

$$
\max _{i} \sum_{j=1}^{I}\left|\{\mathbf{P}\}_{i, j}\right| \equiv \max _{i} \sum_{j=1}^{I}\{\mathbf{P}\}_{i, j} .
$$

From Eq.(II.3) we get

$$
\begin{aligned}
\max _{i} \sum_{j=1}^{J_{\psi}}\{\mathbf{P}\}_{i, j} & =\left.\left.\frac{1}{4 \pi} \max _{i} \sum_{j=1}^{J_{\psi}}\left(-\frac{r_{i}^{2}-R^{2}}{r_{i}}\right) \alpha \ell\left(r_{i}, \psi, R\right)^{-1}\right|_{\alpha=0} ^{2 \pi}\right|_{\psi=\Delta \psi_{j}} \\
& =\left.\frac{1}{2} \max _{i}\left(-\frac{r_{i}^{2}-R^{2}}{r_{i}}\right) \ell\left(r_{i}, \psi, R\right)^{-1}\right|_{\psi=0} ^{\psi_{o}},
\end{aligned}
$$

where $J_{\psi}$ stands for the number of the discretisation steps $\Delta \psi$ within the near zone $\psi \in\left\langle 0, \psi_{o}\right\rangle$. From Eq.(10), the upper bound of the eigenvalues $\lambda_{P}$ is estimated

$$
\max \lambda_{P} \leq \frac{1}{2} \max _{i}\left(-\frac{r_{i}^{2}-R^{2}}{r_{i}}\right)\left[\ell\left(r_{i}, \psi_{o}, R\right)^{-1}-H_{i}^{-1}\right] .
$$

Expressing

$$
\begin{gathered}
\{\mathbf{P}\}_{i, i}-\sum_{\substack{j=1 \\
j \neq i}}^{J_{\psi}}\left|\{\mathbf{P}\}_{i, j}\right| \equiv\{\mathbf{P}\}_{i, i}-\sum_{\substack{j=1 \\
(j \neq i)}}^{J_{\psi}}\{\mathbf{P}\}_{i, j} \equiv 2\{\mathbf{P}\}_{i, i}-\sum_{j=1}^{J_{\psi}}\{\mathbf{P}\}_{i, j} \\
=\frac{1}{4 \pi}\left[-\left.\left.2 \frac{r_{i}^{2}-R^{2}}{r_{i}} \alpha \ell\left(r_{i}, \psi, R\right)^{-1}\right|_{\alpha=0} ^{2 \pi}\right|_{\psi=0} ^{\Delta \psi}+\left.\left.2 \frac{r_{i}^{2}-R^{2}}{r_{i}} \alpha \ell\left(r_{i}, \psi, R\right)^{-1}\right|_{\alpha=0} ^{2 \pi}\right|_{\psi=0} ^{\psi_{o}}\right],
\end{gathered}
$$

the lower bound of the eigenvalues $\lambda_{P}$ is estimated to be

$$
\min \lambda_{P} \geq \frac{1}{2} \min _{i}\left(-\frac{r_{i}^{2}-R^{2}}{r_{i}}\right)\left[2 \ell\left(r_{i}, \Delta \psi, R\right)^{-1}-H_{i}^{-1}-\ell\left(r_{i}, \psi_{o}, R\right)^{-1}\right] .
$$

With reference to the analytical expression in Eq.(II.4), the upper and lower bounds of the eigenvalues $\lambda_{K}$ of the matrix $\mathbf{K}$ for the direct gravity disturbance inversion, see Eq.(3), are estimated from 


$$
\begin{gathered}
\left.\max \lambda_{K} \leq \max _{i} \frac{r_{i}}{2}\left[\sum_{j=1}^{J_{\psi}}\left|-\left(1+\frac{R^{2}}{r_{i}^{2}}\right) \ell\left(r_{i}, \psi, R\right)^{-1}+\frac{r_{i}^{2}-R^{2}}{r_{i}} \frac{r_{i}-R \cos \psi}{\ell\left(r_{i}, \psi, R\right)^{3}}\right|_{\psi=\Delta \psi_{j}}\right]\right], \\
\min \lambda_{K} \geq \min _{i} \frac{r_{i}}{2}\left[-\left(1+\frac{R^{2}}{r_{i}^{2}}\right) \ell\left(r_{i}, \psi, R\right)^{-1}+\left.\frac{r_{i}^{2}-R^{2}}{r_{i}} \frac{r_{i}-R \cos \psi}{\ell\left(r_{i}, \psi, R\right)^{3}}\right|_{\psi=0} ^{\Delta \psi} \mid\right] \\
\left.-\sum_{j=2}^{J_{\psi}}\left|-\left(1+\frac{R^{2}}{r_{i}^{2}}\right)\right| \ell\left(r_{i}, \psi, R\right)^{-1}+\left.\frac{r_{i}^{2}-R^{2}}{r_{i}} \frac{r_{i}-R \cos \psi}{\ell\left(r_{i}, \psi, R\right)^{3}}\right|_{\psi=\Delta \psi_{j}} \mid\right]
\end{gathered}
$$

The upper and lower bounds of the eigenvalues $\lambda_{M}$ of the matrix $\mathbf{M}$ for the direct gravity anomaly inversion, see Eq.(4), are estimated from

$$
\begin{gathered}
\max \lambda_{M} \leq \max _{i} \frac{r_{i}}{2} \sum_{j=1}^{J_{\psi}}\left|-\left(3-\frac{R^{2}}{r_{i}^{2}}\right) \ell\left(r_{i}, \psi, R\right)^{-1}+\frac{r_{i}^{2}-R^{2}}{r_{i}} \frac{r_{i}-R \cos \psi}{\ell\left(r_{i}, \psi, R\right)^{3}}\right|_{\psi=\Delta \psi_{j}} \mid, \\
\min \lambda_{M} \geq \min _{i} \frac{r_{i}}{2}\left[-\left(3-\frac{R^{2}}{r_{i}^{2}}\right) \ell\left(r_{i}, \psi, R\right)^{-1}+\left.\frac{r_{i}^{2}-R^{2}}{r_{i}} \frac{r_{i}-R \cos \psi}{\ell\left(r_{i}, \psi, R\right)^{3}}\right|_{\psi=0} ^{\Delta \psi}\right. \\
\left.-\sum_{j=2}^{J_{\psi}}\left|-\left(3-\frac{R^{2}}{r_{i}^{2}}\right) \ell\left(r_{i}, \psi, R\right)^{-1}+\frac{r_{i}^{2}-R^{2}}{r_{i}} \frac{r_{i}-R \cos \psi}{\ell\left(r_{i}, \psi, R\right)^{3}}\right|_{\psi=\Delta \psi_{j}} \mid\right]
\end{gathered}
$$

\section{NUMERICAL ANALYSIS OF CONDITIONALITY}

The estimation of the upper and lower bounds of the eigenvalues $\lambda_{P}$ of the matrix $\mathbf{P}$, see Eqs.(11) and (13), are shown in Figs. 1 and 2. The upper bound of $\lambda_{P}$ depends on the minimal height $\min H$ within the computation area, whereas the lower bound of $\lambda_{P}$ is functionally related to the discretisation step $\Delta \psi$ and the maximal height max $H$. It follows from Eq.(8) that $\lambda_{P} \leq 1$ when $r_{i} \geq R$ for all $i$. From the estimation of the lower bound of the eigenvalues $\lambda_{P}$ in Eq.(13), the matrix $\mathbf{P}$ may become ill-posed or singular when the discretisation step $\Delta \psi$ coincides with the maximal height max $H$ of the data points by means of $R \Delta \psi \approx \sqrt{3} \max H$, see Fig. 2. In this case, the upper estimate of the condition number $\aleph_{P}$ is infinity large, see Fig. 3 . 


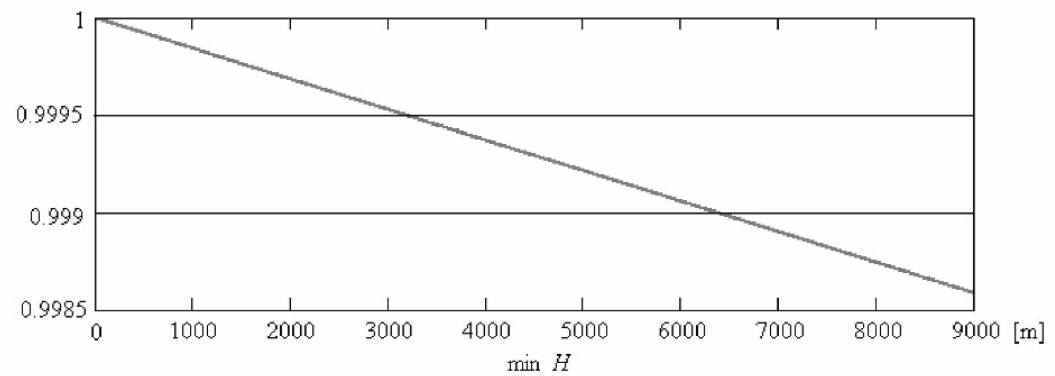

Fig. 1. Estimation of the upper bound of the eigenvalues $\lambda_{P}$ with respect to the lower limit of heights $\min H$.

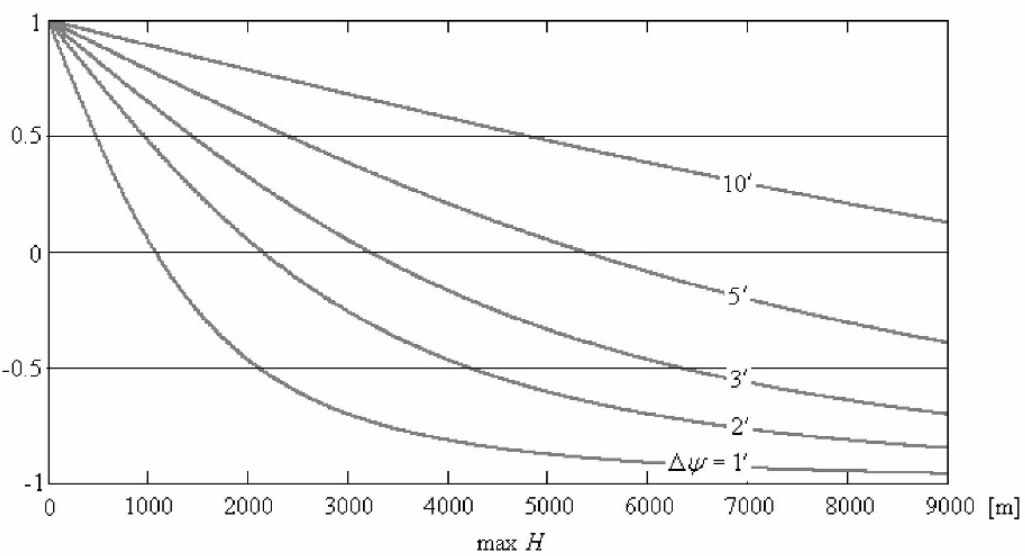

Fig. 2. Estimation of the lower bound of the eigenvalues $\lambda_{P}$ with respect to the upper limit of heights $\max H$ and the discretisation step $\Delta \psi$.

The estimations of the upper and lower bounds of the eigenvalues $\lambda_{K}$ of the matrix $\mathbf{K}$ for the direct gravity disturbance inversion are shown in Figs. 4 and 5. They depend on the minimal height $\min H$ and the maximal height $\max H$ respectively, both correlated with the discretisation step $\Delta \psi$, cf. Eqs.(14) and (15). From Figs. 6 and 7, the upper and lower bounds of the eigenvalues for the direct inversion of gravity anomalies and gravity disturbances are almost identical. Consequently, the upper estimates of the condition numbers $\boldsymbol{\aleph}_{K}$ and $\boldsymbol{\aleph}_{M}$ of the matrices $\mathbf{K}$ and $\mathbf{M}$ are very similar. The upper estimates of the condition numbers $\aleph_{K}$ and $\aleph_{M}$ are correlated with the ratio of the extreme heights $\max H$ and $\min H$ of the data points and the discretisation step $\Delta \psi$. Specifically, the stability of direct gravity inversion may improve with the decreasing range of the heights of data points and increasing discretisation step. It implies that if gravity data are 
distributed at the similar elevations (air-born gravity and ground gravity measurements over flat regions) with the discretisation step $\Delta \psi \geq 1 \mathrm{arcmin}$, the direct gravity inversion is very stable. A theoretical estimation of the conditionality of the direct inversion of ground gravity data can be done based on Fig. 8. The upper estimate of the condition number is $\aleph_{M}<13$ for $\max H<9 \mathrm{~km}$ and $\Delta \psi=1 \operatorname{arcmin}$, while $\aleph_{M}<7$ for $\Delta \psi>2 \operatorname{arcmin}$. Note that the upper bounds of the eigenvalues $\lambda_{M}$ have been estimated for $\min H=100 \mathrm{~m}$. From the upper estimates of the condition numbers $\aleph_{K}$ and $\boldsymbol{\aleph}_{M}$, the direct gravity inversion is sufficiently stable for most of the terrain if the discretization step $\Delta \psi \geq 1$ arcmin is used. If needed, particularly for very detailed gravity data in mountainous regions, the regularization should be applied.

The above theoretical conclusions for the conditionality of the downward continuation and of the direct gravity inversion were further verified by the numerical experiment using the real data. The eigenvalue spectrum was computed by the routine SVDCMP (Press et al. 1992, Chapter 2.6). The computation was conducted at the rough part of the Canadian Rocky Mountains bounded by the parallels of 49 and 52 arcdeg northern geodetic latitude and meridians of 240 and 245 arcdeg eastern geodetic longitude. In our test, the regular geographical data grids of 5 and 2.5 arcmin have been used and the near-zone sub-domain up to 1 arcdeg of the spherical distance has been considered. Over the area of study the mean orthometric heights range from 414 up to $2671 \mathrm{~m}$, see Fig. 9. For the geographical data grid of 5 arcmin, which approximately corresponds in terms of $\Delta \psi=(\Delta \lambda / 2) \cos \phi$ to the discretisation step $\Delta \psi \approx 2.5$ arcmin, we obtained the condition number $\aleph_{P}$ of 2.3 for the downward continuation and corresponding value $\aleph_{M}$ of 1.5 for the direct gravity inversion. Consequently, for the geographical data grid of $2.5 \mathrm{arcmin}$, i.e., $\Delta \psi \approx 1.2$ arcmin, we obtained $\aleph_{P}$ of 2.7 and $\aleph_{M}$ of 2.45 . These numerical results are in

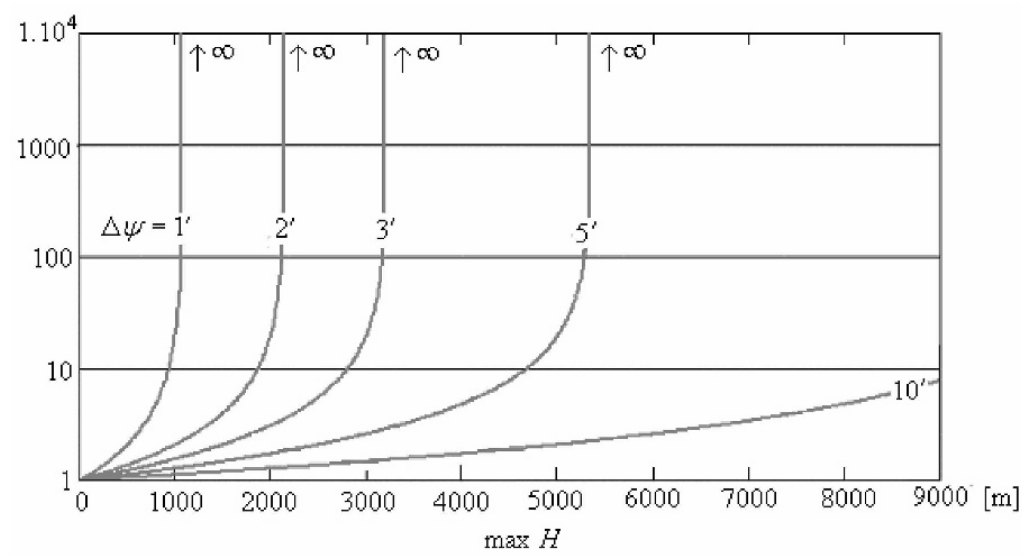

Fig. 3. Upper estimate of the condition number $\aleph_{P}$ with respect to the upper limit of heights $\max H$ and the discretisation step $\Delta \psi$. 


\section{Conditionality of Inverse Solutions to Discretised Integral Equations in Geoid Modelling ...}

good agreement with the upper estimates of the condition numbers from Figs. 3 and 8, except for the downward continuation using 2.5 arcmin data grid. In contrary to the theoretical expectation, the stability of the downward continuation improves after reaching the critical value $R \Delta \psi \approx \sqrt{3} \max H$, see Fig. 3 .

The numerical experiment using the real data reveals a better stability of the direct gravity inversion. Such a conclusion can be deduced also from a comparison of the AbelPoisson kernel $\mathcal{P}$ for the downward continuation, see Eq.(I.3), with the Green integral kernels $\mathcal{J}$ and $\mathcal{M}$ for the direct gravity inversion, see Eqs.(I.5) and (I.7); see also Figs. 10a,b,c. Note that the kernels are scaled with respect to their maximal values. Since

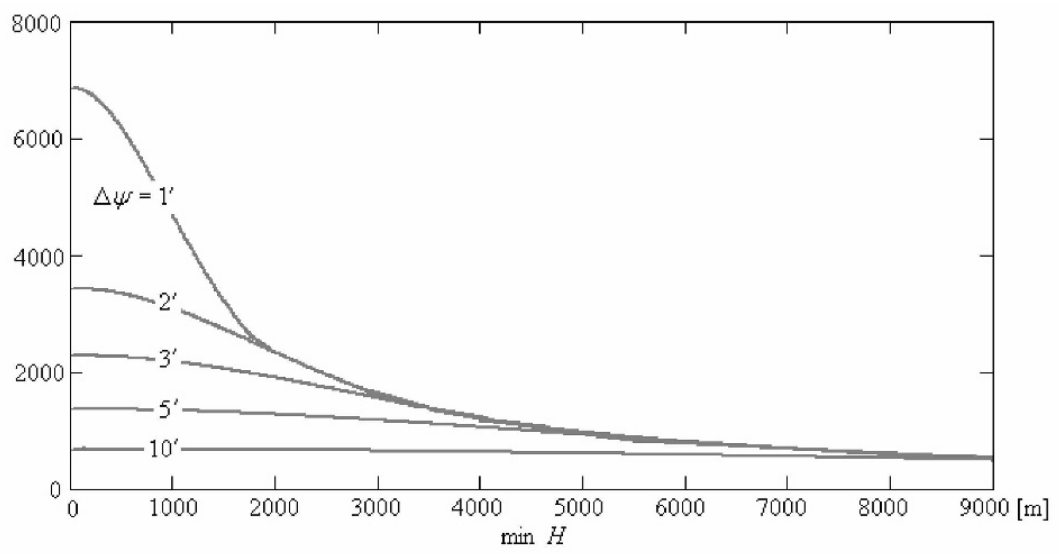

Fig. 4. Estimation of the upper bound of the eigenvalues $\lambda_{K}$ with respect to the lower limit of heights $\min H$ and the discretisation step $\Delta \psi$.

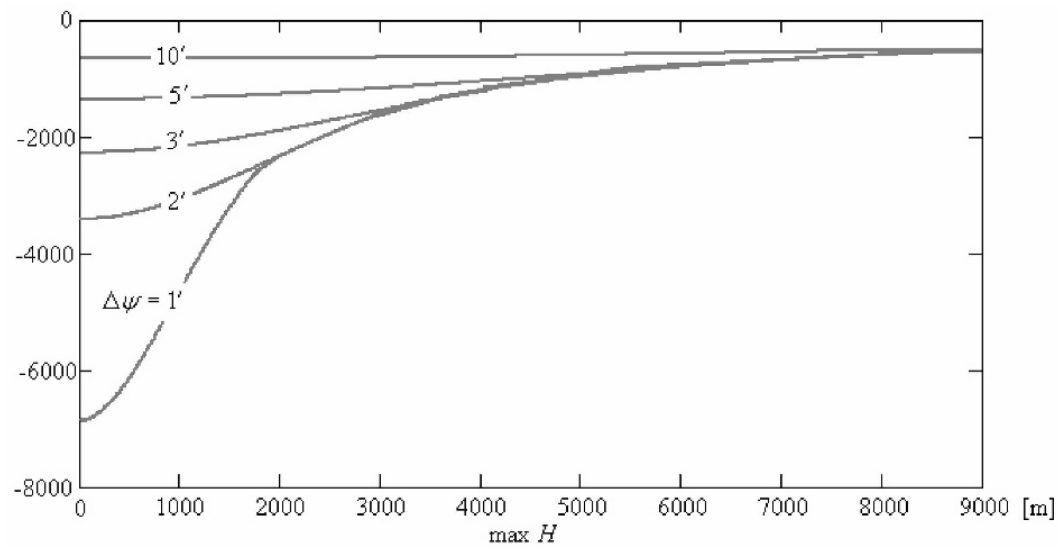

Fig. 5. Estimation of the lower bound of the eigenvalues $\lambda_{K}$ with respect to the upper limit of heights max $H$ and the discretisation step $\Delta \psi$. 
$\mathcal{J}$ and $\mathcal{M}$ descending more quickly with $\psi$ than $\mathcal{P}$, the contributions of the off-diagonal elements $\sum_{\substack{j=1 \\ j \neq i}}^{I}\left|\{\mathbf{K}\}_{i, j}\right|$ and $\sum_{\substack{j=1 \\ j \neq i}}^{I}\left|\{\mathbf{M}\}_{i, j}\right|$ in $\mathbf{K}$ and $\mathbf{M}$ respectively are smaller than the corresponding contribution $\sum_{\substack{j=1 \\ j \neq i}}^{I}\left|\{\mathbf{P}\}_{i, j}\right|$ in $\mathbf{P}$.

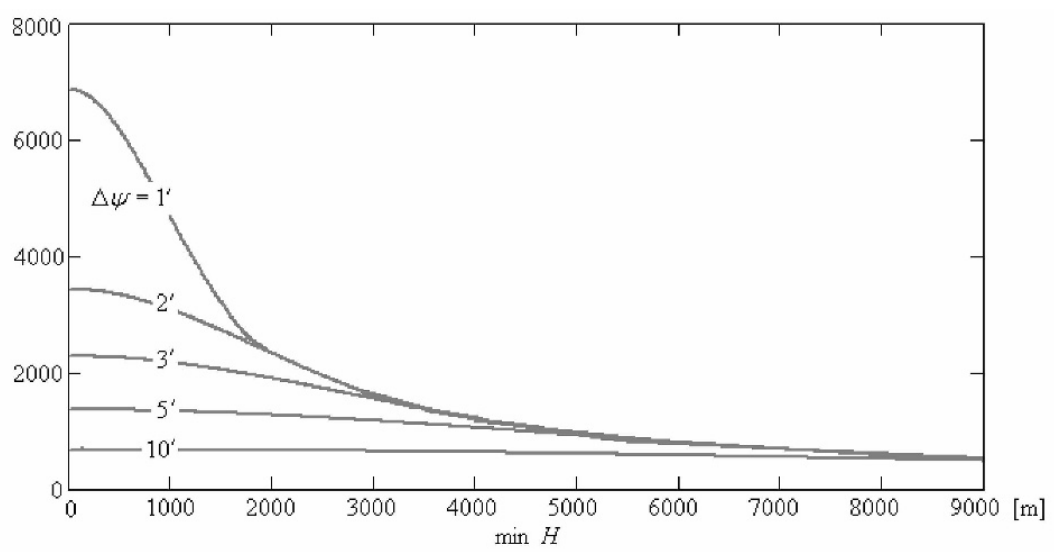

Fig. 6. Estimation of the upper bound of the eigenvalues $\lambda_{M}$ with respect to the lower limit of heights $\min H$ and the discretisation step.

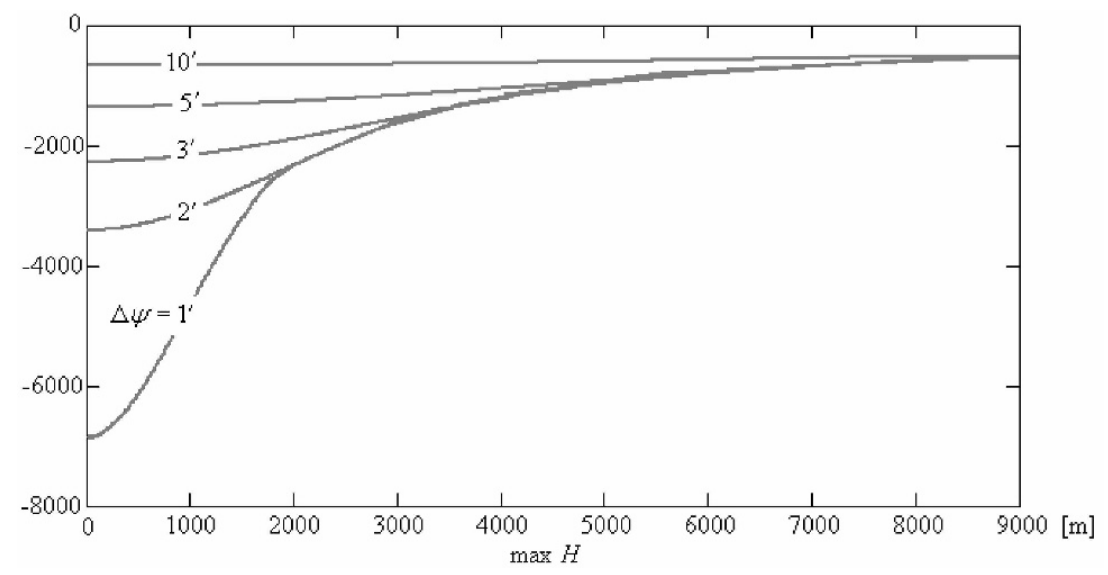

Fig. 7. Estimation of the lower bound of the eigenvalues min $\lambda_{M}$ with respect to the upper limit of heights max $H$ and the discretisation step $\Delta \psi$. 


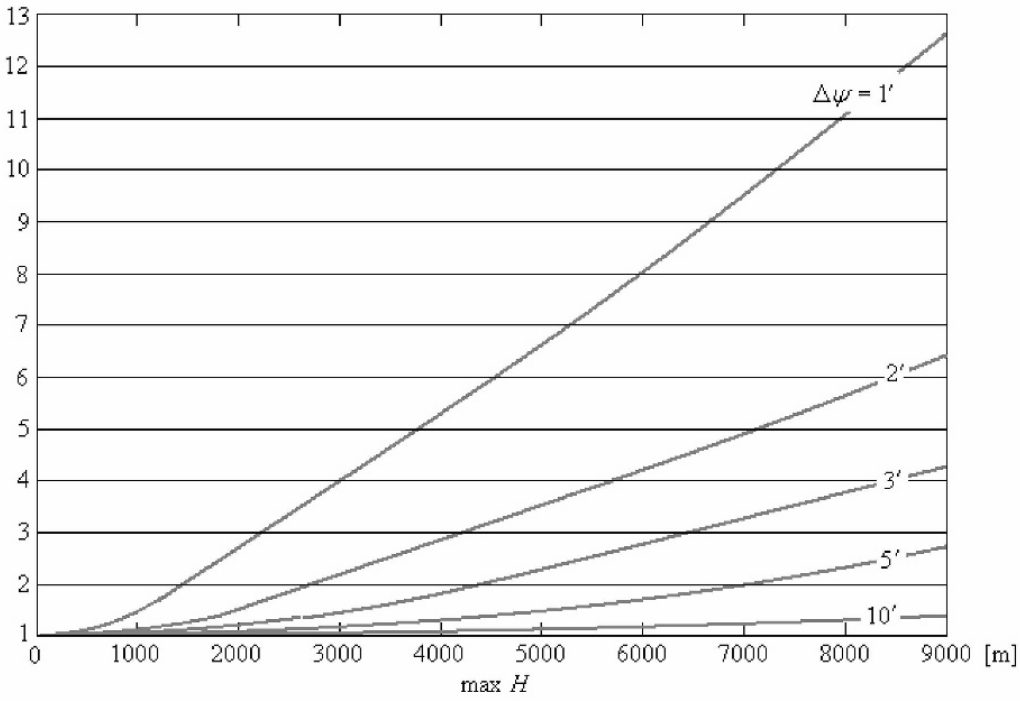

Fig. 8. Upper estimates of the condition numbers $\aleph_{M}$ and $\aleph_{K}$ with respect to the upper limit of heights $\max H$ and the discretisation step $\Delta \psi$. The upper bounds of the eigenvalues were estimated for $\min H=100 \mathrm{~m}$.

The influence of the size of the computation area on the upper estimates of the condition numbers was finally investigated. The results from Figs. 11 and 12 ascertain that the far-zone influence $\psi \in\left(\psi_{o}, \pi\right\rangle$ for $\psi_{o}>1$ arcdeg and the discretisation step 1 arcmin is not significant on the conditionality property of the inverse solutions. For the increasing area of the near-zone sub-domain, the upper estimate of the condition number for the direct gravity inversion slightly decreases. In contrary, the upper estimation of the condition number for the downward continuation slightly increases. Note that such statements hold only if the extreme heights of data points remain unchanged with reduction of the computation area.

\section{CONCLUSIONS}

The conditionality of inverse solutions to the two alternative computational models for determination of the disturbing gravity potential from local gravity data has been analysed. The parameterisation of the solution has been considered so that its horizontal distribution is identical with the regular grid of the data points to preserve the predominant structure of the matrices for inversion. The eigenvalue decomposition technique has been applied for the analysis of conditionality. The numerical experiments with the real data reveal that the direct gravity inversion is more stable than the downward continuation. Finally, from upper estimates of the condition numbers we acquired that the conditionality of inverse solutions is not significantly affected by the far-zone effects (if $\psi_{o}>1 \operatorname{arcdeg}$ ). 


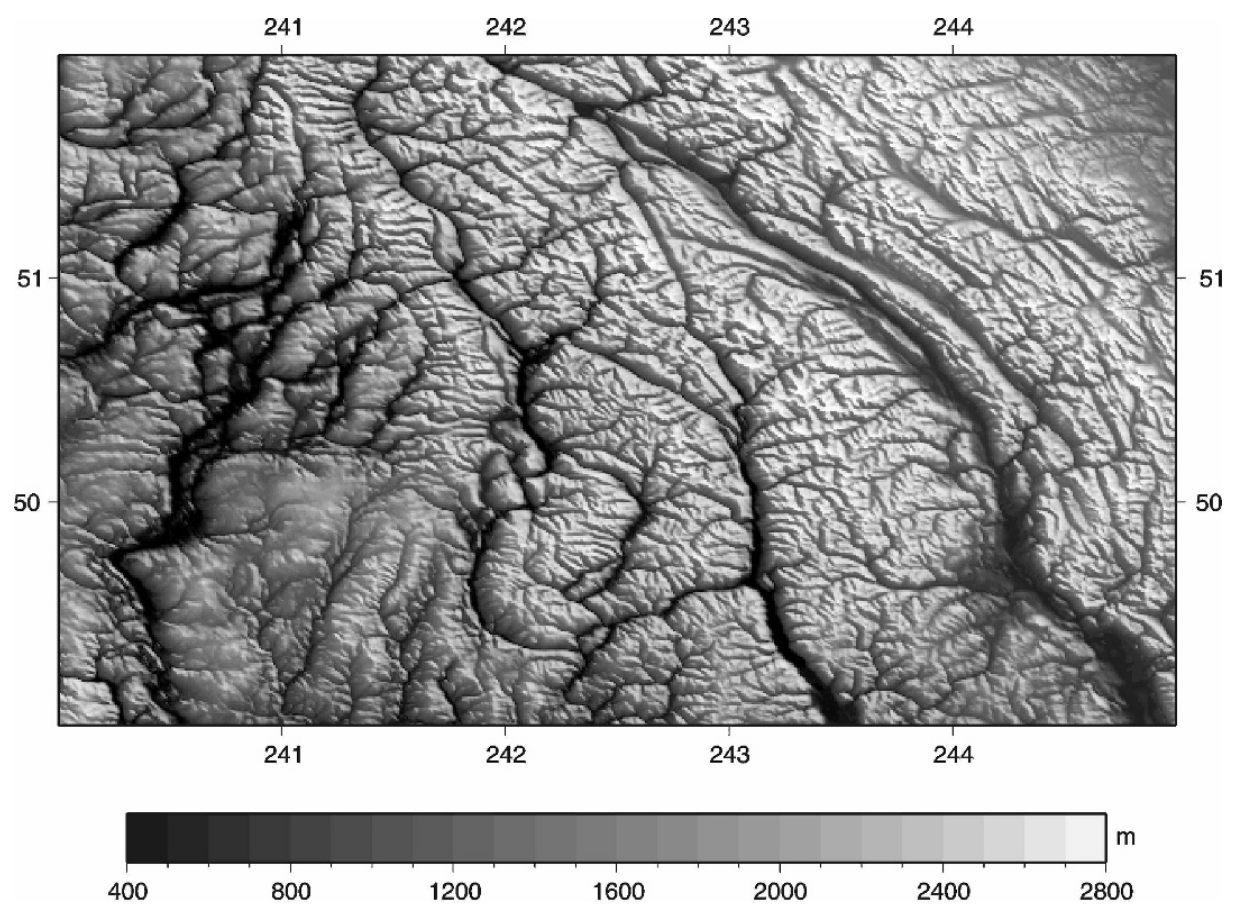

Fig. 9. Topography at the study area (m).

Concluding from our results, the direct gravity inversion is more appropriate than the standard two-step approach, which utilises the inverse Abel-Poisson integral equation and consequently the Stokes/Hotine integration. Despite remaining high-frequency errors inherent in the downward continuation are further reduced or eliminated after applying the Stokes/Hotine integration, the downward continuation requires more complex regularization. This is the case especially in mountainous areas with an available detailed local gravity data. Due to a smoothing effect of the regularisation, however, some information about the detailed gravity signal can be lost. Since lower requirements on the regularization, the direct gravity inversion is thus advisable. Another advantage of the direct gravity inversion is its better numerical efficiency. 
Conditionality of Inverse Solutions to Discretised Integral Equations in Geoid Modelling ...

a)

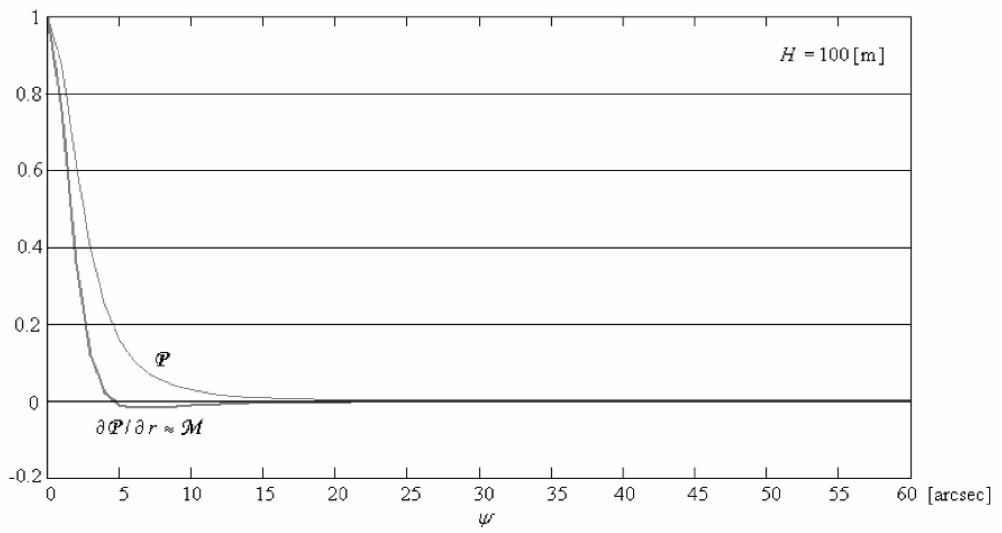

b)

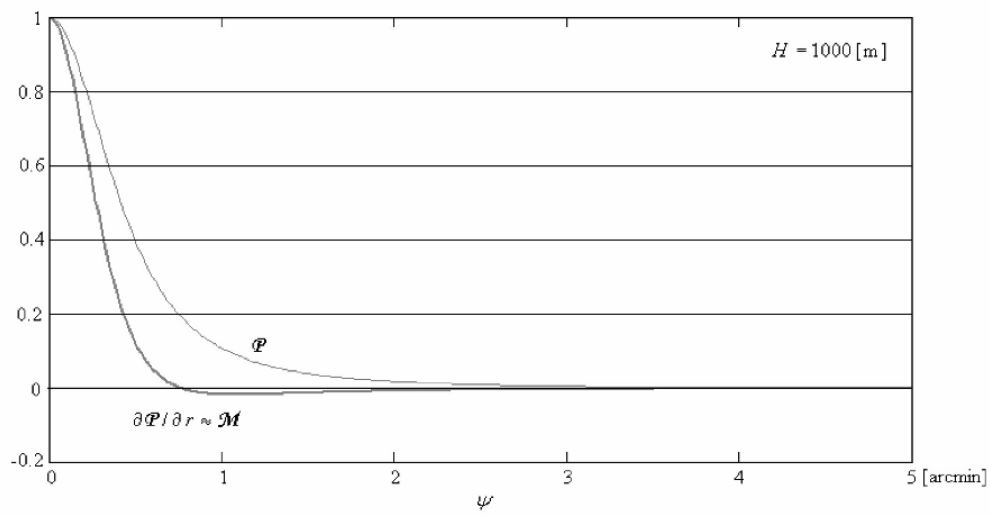

c)

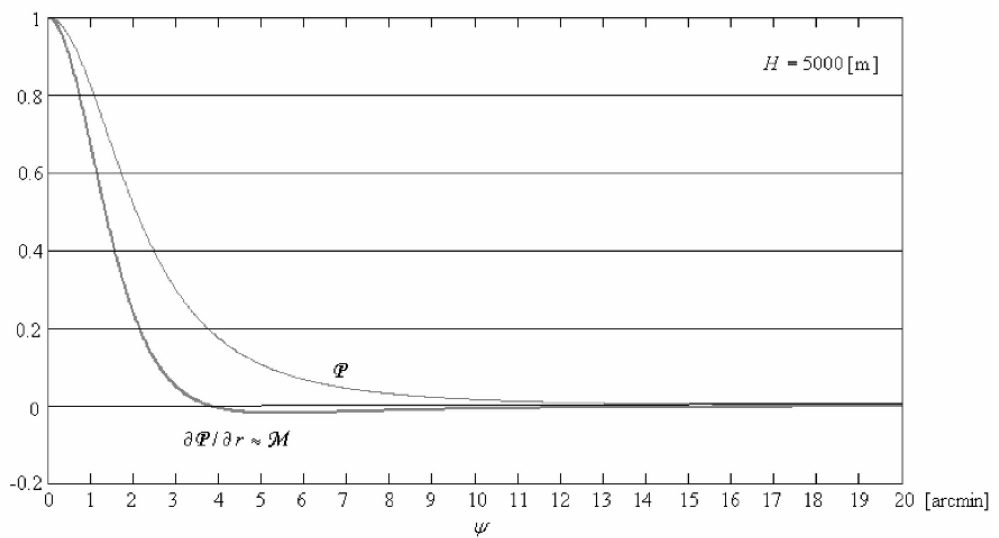

Fig. 10. Integral kernels $\mathcal{P}, \mathcal{J}=\partial \mathcal{P} / \partial r$ and $\mathcal{M}$ scaled with respect to their maximal values: a) $H=100 \mathrm{~m}$, interval $\psi \in\langle 0,1\rangle$ arcmin; b) $H=1000 \mathrm{~m}$, interval $\psi \in\langle 0,5\rangle \operatorname{arcmin}$; c) $H=5000 \mathrm{~m}$, interval $\psi \in\langle 0,20\rangle$ arcmin. Note that the integral kernels $\mathcal{J}=\partial \mathcal{P} / \partial r$ and $\mathcal{M}$ are practically identical. 


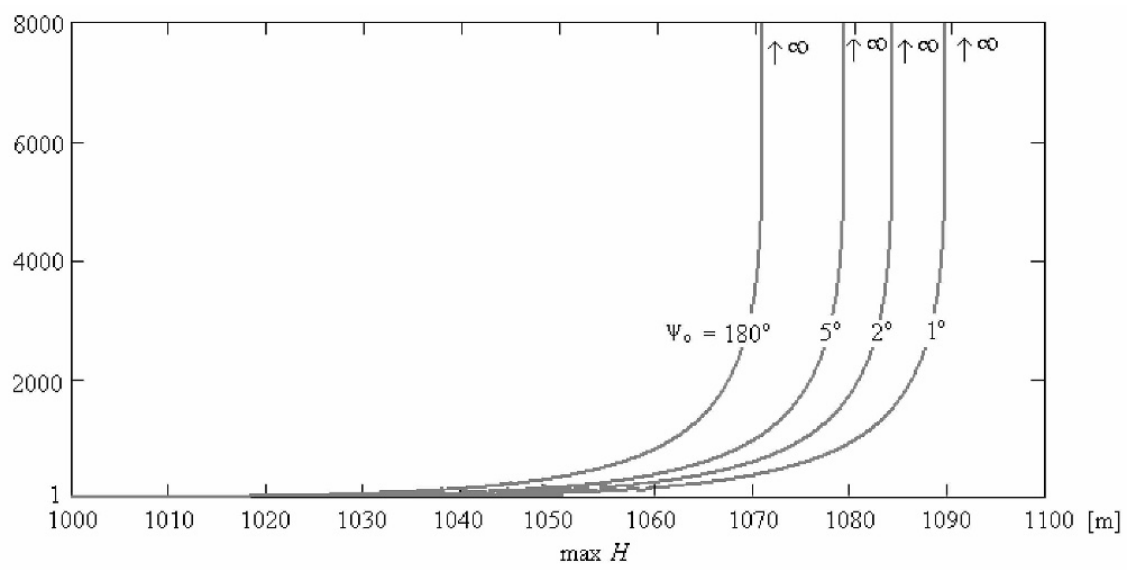

Fig. 11. Influence of the far-zone on the upper estimate of the condition number $\aleph_{P}$. The upper estimates of the condition number $\aleph_{P}$ are given for the near zone integration areas of $\psi_{o}=1,2,5$ arcdeg and for the full integration domain, i.e., $\psi \in\langle 0, \pi\rangle$. The discretisation step $\Delta \psi$ of 1 arcmin was used.

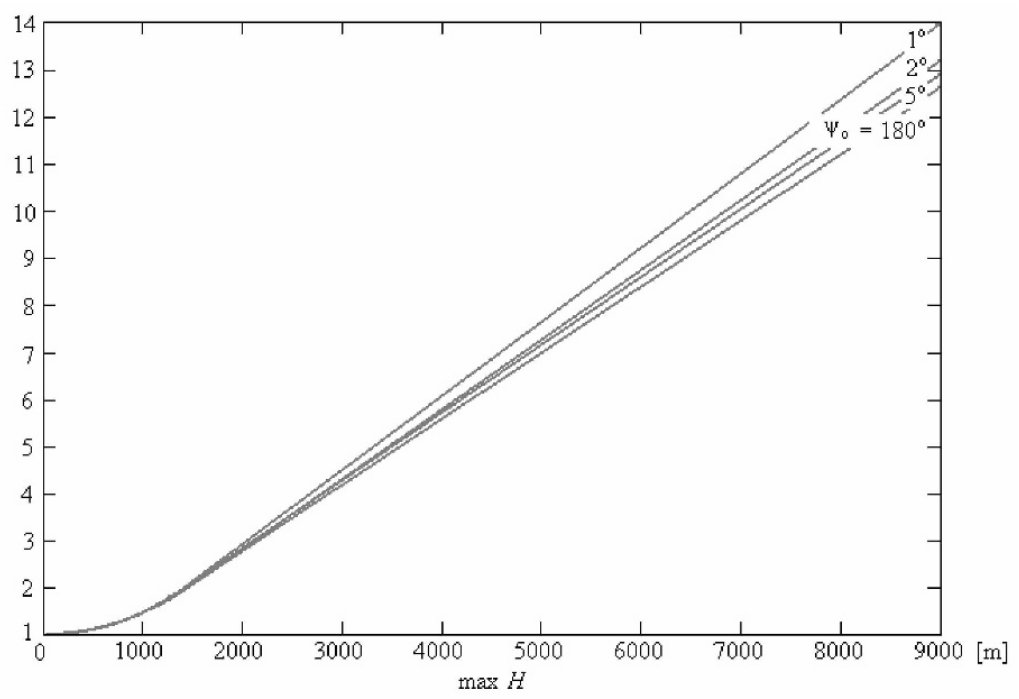

Fig. 12. Influence of the far-zone on the upper estimate of the condition number $\boldsymbol{\aleph}_{M}$. The upper estimates of the condition number $\boldsymbol{\aleph}_{M}$ are given for the near zone integration areas of $\psi_{\mathrm{o}}=1,2,5$ arcdeg and for the full integration domain, i.e., $\psi \in\langle 0, \pi\rangle$. The discretisation step $\Delta \psi$ of 1 arcmin was used. 


\section{APPENDIX I. \\ GREEN'S INTEGRALS FOR UPWARD CONTINUATION AND DIRECT GRAVITY INVERSION}

After linearization and spherical approximation, the harmonic upward continuation of the gravity disturbance $\delta g$ and the gravity anomaly $\Delta g$, both multiplied by the geocentric radius $\boldsymbol{r}$, is defined by the Abel-Poisson integral (see e.g., Kellogg, 1929)

$$
\begin{aligned}
& r \delta g(r, \Omega)=\frac{1}{4 \pi} \iint_{\Omega^{\prime} \in \Omega_{O}} \mathcal{P}(r, \psi, R) R \delta g\left(R, \Omega^{\prime}\right) d \Omega^{\prime}, \forall r>R: \nabla^{2} r \delta g(r, \Omega)=0, \\
& r \Delta g(r, \Omega)=\frac{1}{4 \pi} \iint_{\Omega^{\prime} \in \Omega_{O}} \mathcal{P}(r, \psi, R) R \Delta g\left(R, \Omega^{\prime}\right) d \Omega^{\prime}, \forall r>R: \nabla^{2} r \Delta g(r, \Omega)=0,
\end{aligned}
$$

where $\Omega_{O}$ is the total solid angle, and $d \Omega^{\prime}=\cos \phi^{\prime} d \phi^{\prime} d \lambda^{\prime}$ the infinitesimal surface element on the unit sphere. The spherical Abel-Poisson kernel $\mathcal{P}$ reads (ibid.)

$$
\begin{aligned}
\forall r>R: \quad & \mathcal{P}(r, \psi, R)=\sum_{n=2}^{\infty}(2 n+1)\left(\frac{R}{r}\right)^{n+1} P_{n}(\cos \psi) \\
& =R \frac{r^{2}-R^{2}}{\ell(r, \psi, R)^{3}}-\frac{R}{r}-3 \frac{R^{2}}{r^{2}} \cos \psi .
\end{aligned}
$$

where $P_{n}(\cos \psi)$ are the Legendre polynomials (e.g., Hobson, 1931) for the argument of cosine of the spherical angle $\psi$ of two points $(r, \Omega)$ and $\left(R, \Omega^{\prime}\right)$, and $\ell$ is their corresponding Euclidean (spatial) distance. The geocentric latitude $\phi$ and longitude $\lambda$ represent the geocentric direction $\Omega=(\phi, \lambda)$, and $r$ is the geocentric radius. The mean radius of the Earth $R$ is adopted in a spherical approximation of the geocentric radius $r_{g}$ of the geoid, i.e., $r_{g} \approx R$.

The gravity disturbance $\delta g$ is evaluated by integral convolution of the radiallydifferentiated Abel-Poisson kernel and the disturbing gravity potential $T$ on the geoid, $\forall r>R: \nabla^{2} T(r, \Omega)=0$; see Novák (2003)

$$
r \delta g(r, \Omega) \cong-r \frac{\partial T(r, \Omega)}{\partial r}=-\frac{r}{4 \pi} \iint_{\Omega^{\prime} \in \Omega_{O}} \mathcal{J}(r, \psi, R) T\left(R, \Omega^{\prime}\right) d \Omega^{\prime},
$$

where the integral kernel $\mathcal{I}$ reads (ibid.) 


$$
\begin{gathered}
\forall r>R: \quad \mathcal{J}(r, \psi, R)=\frac{\partial \mathcal{P}(r, \psi, R)}{\partial r}=-\sum_{n=2}^{\infty}(2 n+1)\left(\frac{R}{r}\right)^{n+1} \frac{n+1}{r} P_{n}(\cos \psi) \\
=\frac{2 r R}{\ell(r, \psi, R)^{3}}-3 R \frac{r^{2}-R^{2}}{\ell(r, \psi, R)^{5}}(r-R \cos \psi)+\frac{R}{r^{2}}+6 \frac{R^{2}}{r^{3}} \cos \psi .
\end{gathered}
$$

By analogy with Eq.(I.4), for the gravity anomaly $\Delta g$ we get (ibid.)

$$
r \Delta g(r, \Omega) \cong-r\left[\frac{\partial T(r, \Omega)}{\partial r}+\frac{2}{r} T(r, \Omega)\right]=-\frac{r}{4 \pi} \iint_{\Omega^{\prime} \in \Omega_{O}} \mathcal{M}(r, \psi, R) T\left(R, \Omega^{\prime}\right) d \Omega^{\prime} .
$$

The integral kernel $\mathcal{M}$ in Eq.(I.6) linearly combines $\mathcal{P}$ and $\mathcal{J}$; i.e.,

$$
\mathcal{M}(r, \psi, R)=\mathcal{J}(r, \psi, R)+\frac{2}{r} \mathcal{P}(r, \psi, R),
$$

whence (ibid.)

$$
\begin{gathered}
\forall r>R: \quad \mathcal{M}(r, \psi, R)=-\sum_{n=2}^{\infty}(2 n+1) \frac{n-1}{r}\left(\frac{R}{r}\right)^{n+1} P_{n}(\cos \psi) \\
\quad=-3 R \frac{r^{2}-R^{2}}{\ell(r, \psi, R)^{5}}(r-R \cos \psi)+\frac{2 R}{r}+\frac{2 r^{2}-R^{2}}{\ell(r, \psi, R)^{3}}-\frac{R}{r^{2}}
\end{gathered}
$$

\section{APPENDIX II.}

\section{ANALYTICAL SOLUTION OF GREEN'S INTEGRALS FOR THE DIRECT GRAVITY INVERSION IN TERMS OF THE POLAR SPHERICAL COORDINATES}

With reference to Eq.(I.6), the discretised Green integrals for the direct gravity anomaly inversion in terms of the polar spherical coordinates $\psi$ and $\alpha$ reads

$$
r \Delta g(r, \Omega) \cong-\left.\left.\frac{r}{4 \pi} \sum_{j=1}^{J} T\left(R, \Omega_{j}\right)\left(\frac{\partial \mathrm{F}_{\mathcal{P}}\left(r, \psi_{j}, R\right)}{\partial r}+\frac{2}{r} F_{\mathcal{P}}\left(r, \psi_{j}, R\right)\right)\right|_{\Delta \alpha_{j}}\right|_{\Delta \psi_{j}}
$$

where $T\left(R, \Omega_{j}\right)$ are the disturbing gravity potential values parameterised for the geometrical centres $\left(R, \Omega_{j}\right)$ of the discretised surface elements. The discretisation steps with respect to the spherical azimuth and spherical distance are denoted $\Delta \alpha$ and $\Delta \psi$ respectively. $J$ is the number of discrete surface elements on the unit sphere $\Omega_{O}$. From the expression for $\partial \ell^{-1} / \partial \psi$, given by 


$$
\frac{\partial \ell(r, \psi, R)^{-1}}{\partial \psi}=-\frac{r R \sin \psi}{\ell^{3}(r, \psi, R)},
$$

the analytical solution for the Abel-Poisson integral is found to be

$$
\begin{gathered}
\left.\left.F_{\mathcal{P}}(r, \psi, R)\right|_{\Delta \alpha}\right|_{\Delta \psi}=\int_{\Delta \alpha \Delta \psi} \int_{\Delta} \mathcal{P}(r, \psi, R) \sin \psi d \psi d \alpha \\
=\left.R\left(r^{2}-R^{2}\right) \alpha \int_{\Delta \psi} \ell(r, \psi, R)^{-3} \sin \psi d \psi\right|_{\Delta \alpha}=-\left.\left.\frac{r^{2}-R^{2}}{r} \alpha \ell(r, \psi, R)^{-1}\right|_{\Delta \alpha}\right|_{\Delta \psi} .
\end{gathered}
$$

The differentiation of Eq.(II.3) with respect to $r$ yields the analytical solution of the Green integral for the direct gravity disturbance inversion in Eq.(I.4); i.e.,

$$
\begin{aligned}
& r \delta g(r, \Omega) \cong-\left.\left.\frac{r}{4 \pi} \sum_{j=1}^{J} T\left(R, \Omega_{j}\right) \frac{\partial F_{\mathcal{P}}\left(r, \psi_{j}, R\right)}{\partial r}\right|_{\Delta \alpha_{j}}\right|_{\Delta \psi_{j}}=-\frac{r}{4 \pi} \sum_{j=1}^{I} T\left(R, \Omega_{j}\right) \\
& \times\left.\left.\alpha\left[-\left(1+\frac{R^{2}}{r^{2}}\right) \ell(r, \psi, R)^{-1}+\frac{r^{2}-R^{2}}{r} \frac{r-R \cos \psi}{\ell(r, \psi, R)^{3}}\right]\right|_{\Delta \alpha_{j}}\right|_{\Delta \psi_{j}} .
\end{aligned}
$$

Substitution from Eqs.(II.3) and (II.4) to Eq.(II.1) finally yields

$$
\begin{aligned}
r \Delta g(r, \Omega) \cong & -\frac{r}{4 \pi} \sum_{j=1}^{J} T\left(R, \Omega_{j}\right) \alpha\left[-\frac{3 r^{2}-R^{2}}{r^{2}} \ell\left(r, \psi_{j}, R\right)^{-1}\right. \\
& \left.+\frac{r^{2}-R^{2}}{r} \frac{r-R \cos \psi_{j}}{\ell\left(r, \psi_{j}, R\right)^{3}}\right]\left.\left.\right|_{\Delta \alpha_{j}}\right|_{\Delta \psi_{j}} .
\end{aligned}
$$

Acknowledgments: We thank Dr. P. Holota and Dr. I. Prutkin for their constructive comments. P. Novák was supported by the Czech Science Foundation (Grant 205/05/2777) and the Czech Ministry of Education, Youth and Sport (Research Plans MSM4977751301 and LC506).

\section{References}

Alberts B. and Klees R., 2004. A comparison of methods for the inversion of airborne gravity data. J. Geodesy, 78, 55-65.

Arfken G., 1985. Mathematical Methods for Physicists, 3rd Edition. Academic Press, Orlando.

Bjerhammar A., 1962. Gravity Reductions to a Spherical Surface. Royal Institute of Technology, Division of Geodesy, Stockholm. 
Bjerhammar A., 1963. A New Theory of Gravimetric Geodesy. Royal Institute of Technology, Division of Geodesy, Stockholm.

Bjerhammar A., 1987. Discrete Physical Geodesy. Report 380, Dept. of Geodetic Science and Surveying, The Ohio State University, Columbus.

Engels H., Grafarend E., Keller W., Martinec Z., Sansò F. and Vaníček P., 1993. The geoid as an inverse problem to be regularized. In: G. Anger, R. Gorenflo, H. Jochmann, H. Moritz and W. Webers (Eds.), Inverse Problems: Principles and Applications in Geophysics, Technology and Medicine. Akademie-Verlag, Berlin, 122-167.

Hageman L.and Young D., 1981. Applied Iterative Methods. New York, Academic Press.

Hobson E.W., 1931. The Theory of Spherical and Ellipsoidal Harmonics. Cambridge University Press, Cambridge.

Ilk K.H., 1993. Regularization for high resolution gravity field recovery by future satellite techniques. In: G. Anger, R. Gorenflo, H. Jochmann, H. Moritz and W. Webers (Eds.), Inverse Problems: Principles and Applications in Geophysics, Technology and Medicine. AkademieVerlag, Berlin, 189-214.

Kellogg O.D., 1929. Foundations of Potential Theory. Springer, Berlin.

Lavrentiev M.M., Romanov V.G. and Shishatskii S.P., 1986. Ill-Posed Problems of Mathematical Physics and Analysis. Translated by J.R. Schulenberger, Translations of Mathematical Monographs, 64, American Mathematical Society, Providence, R. I., 1986, ISBN 0-28184517-9.

Martinec Z., 1996. Stability investigations of a discrete downward continuation problem for geoid determination in the Canadian Rocky Mountains. J. Geodesy, 70, 805-828.

Martinec Z., 1998. Boundary value problems for gravimetric determination of a precise geoid. Lecture Notes in Earth Sciences, 73, Springer, Berlin and Heidelberg, Germany.

Novák P., 2000. Evaluation of Gravity Data for the Stokes-Helmert Solution to the Geodetic Boundary-Value Problem. Technical Report 207, UNB, Fredericton, Canada.

Novák P., 2003. Geoid determination using one-step integration. J. Geodesy, 77, 193-206.

Novák P., Kern M., Schwarz K.P., Sideris M.G., Heck B., Ferguson S., Hammada Y. and Wei M., 2003. On geoid determination from airborne gravity. J. Geodesy, 76, 510-522.

Press W.H., Flannery B.P., Teukolsky S.A. and Vetterling W.T., 1992. Numerical Recipes in FORTRAN: The Art of Scientific Computing, 2nd Edition. Cambridge University Press, Cambridge, U.K.

Sideris M.G. and Forsberg R., 1991. Review of geoid prediction methods in mountainous regions. In: R.H. Rapp and F. Sansò (Eds.), Determination of the Geoid, Present and Future. IAG Symposia, 106, Springer-Verlag, Berlin and Heidelberg, 51-62.

Tikhonov A.N., 1963. Regularization of incorrectly posed problems. Soviet Math. Dokl., 4(6), $1624-1627$.

Tikhonov A.N. and Arsenin V.Y., 1977. Solutions of Ill-Posed Problems. V.H. Vinston, Washington, D.C.

Varga R.S., 1962. Matrix Iterative Analysis. Prentice-Hall, Englewood Cliffs, NJ.

Wilkinson J., 1965. The Algebraic Eigenvalue Problem. Oxford University Press, Oxford, U.K.

Young D., 1972. Iterative Solutions of Large Linear Systems. Academic Press, New York. 